\title{
Botulinum toxin A should not be first-line therapy for overactive bladder
}

\author{
Lesley K. Carr, MD, FRCSC
}

Associate Professor, Department of Surgery, University of Toronto, Sunnybrook Health Sciences Centre, Toronto, ON

Cite as: Can Urol Assoc J 201 1;5(3):204-5; D01:10.5489/cuaj.11044

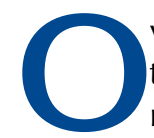

veractive bladder $(O A B)$ is a very prevalent condition in both men and women and has a significant negative impact on quality of life. ${ }^{1}$ It is characterized by urgency to void and is generally associated with frequency of urination and nocturia. The coexistence of urge incontinence, $\mathrm{OAB}$-wet, is more common in women. Overactive bladder may be associated with known neurologic conditions, such as spinal cord injury, multiple sclerosis and stroke (neurogenic $O A B$ ), or may occur in otherwise neurologically intact individuals (idiopathic $O A B$ ).

Traditional management of OAB should start with behavioural therapies (i.e., timed/prompted voiding, urge suppression, modification of type and timing of fluids and pelvic floor rehabilitation). Behavioural therapy has been shown to be effective at reducing urge incontinence alone or when combined with pharmacotherapy. ${ }^{2}$ When behavioural strategies fail to achieve adequate control of OAB symptoms, anticholinergic medications are advocated next. There are a number of agents available; the selection should take into consideration a balance of efficacy and tolerability along with drug benefit coverage, when available. All prescription agents available in Canada have been shown to be effective in reducing $\mathrm{OAB}$ symptoms compared to placebo. While side effects are frequent with this group of medications (dry mouth, constipation, blurry vision amongst the most common), there are very few absolute contraindications: untreated narrow angle glaucoma, obstruction of the gastrointestinal or genitourinary system and myasthenia gravis.

Botulinum toxin A (BTX A) has emerged as a new, effective treatment for refractory neurogenic and idiopathic OAB. Since early promising publications, ${ }^{3-5}$ urologists have used BTX A in Canada for this purpose, but it continues to be done off-label as there is currently no formal approval for BTX A for OAB. Doses of 100 to 200 units of BTX A for idiopathic OAB and 200 to 300 units of BTX A for neu- rogenic OAB have been shown in placebo controlled trials to improve primary and secondary endpoints of urge incontinence episodes, maximum cystometrogram capacity, maximum detrusor pressure and quality of life..$^{4,6-8}$ The procedure is relatively simple and requires only an intravesical instillation of topical anesthetic in most patients. However, injection of BTX A into the detrusor remains an invasive procedure and carries risks of gross hematuria (6\%-10\%) and urinary tract infection $(7 \%-22 \%) ;{ }^{9}$ some patients also experience more bladder pain requiring anesthetic support. A frequent, dose- dependent complication of BTX A detrusor injections is increased post-void residual or urinary retention. The number of patients with idiopathic OAB treated with BTX A who converted to post-void residual related catheterization ranged from $5 \%$ to $21 \% .^{10}$ The side effect of urinary retention may be of little concern for the neurogenic $\mathrm{OAB}$ patient who is already self-catheterizing, but for the idiopathic $O A B$ patient, the potential for intermittent selfcatheterization may prohibit some patients from considering this option. In addition, rare systemic side effects, such as diffuse muscle weakness, have been reported $(1 \%) .{ }^{11}$ The etiology of this very rare complication is not known and it seems to be self-limiting and lasts from weeks to a couple of months. Finally, it is inherently expected that BTX A injections will need to be repeated in 3- to 12-month intervals. Studies have shown that repeated injections are effective, ${ }^{11}$ however, there is theoretical concern that repeated injection may cause antibody formation over time with a loss of efficacy. Factors felt to increase the chance of antibody formation (based on experience with BTX A for cervical dystonia) are felt to be protein load (lower protein load $5 \mathrm{ng} / 100 \mathrm{U}$ in the formulation used since 1987), shorter duration between injections and larger doses. ${ }^{11,12}$ A meta-analysis of published seroconversion estimates the rate of development of antibodies to BTX A for various conditions as follows: cervical dystonia (4 of 312), glabellar lines(2 of 718), OAB (0 of 22) and hyperhydrosis (4 of 871$).{ }^{12}$ 
Currently, the cost of BTX A remains a challenge for many who would benefit from such treatment. Because there is no formal approval for BTX A for bladder indications by Health Canada, many provincial and private drug formularies do not cover the cost of the agent (about $\$ 400$ for 100 units). Cost-effectiveness studies have been done comparing BTX A injections to standard therapy. ${ }^{13,14}$ Data suggest that BTX A injections cost $\$ 14377$ (US) per quality-adjusted life-year gained..$^{13} \mathrm{~A}$ strategy is often considered cost-effective when the incremental cost-effectiveness ratio is less than $\$ 50000$ per quality-adjusted life-year. Another European study found a cost-effectiveness ratio of $617 £$ suggesting that BTX A was highly likely to be cost-effective in both idiopathic and neurogenic OAB.$^{14}$ However, it is important to consider that both of these cost-modelling studies were done for a group of patients who failed an initial attempt with pharmacotherapy. Undoubtedly, if a patient with OAB is successfully managed with behavioural therapy and a single first-line anticholinergic agent, the cost to the health care system is less than repeated treatments with BTX A.

While BTX A has been shown to be more effective at controlling the symptoms of both neurogenic and idiopathic OAB than anticholinergic medications in the subset of patients who are refractory to anticholinergic drugs, there has not been a trial comparing these 2 treatments in patients naïve to either treatment. This information would be relevant if one is considering advocating BTX A as first-line therapy for OAB.

Thus, while the use of intravesical BTX A has been shown to be a safe and effective therapy for refractory neurogenic and idiopathic $O A B$, it should still be reserved for patients who have failed first-line therapy with behavioral and pharmacological therapies.

Competing interests: None declared.

\section{References}

1. Jackson S. The patient with an overactive bladder-symptoms and quality-of-life issues. Urology 1997;50:1822.

2. Burgio KL, Locher JL, Goode PS, et al. Behavioral vs drug treatment for urge urinary incontinence in older women: a randomized controlled trial. JAMA 1998;280:1995-2000.

3. Reitz A, Stohrer M, Kramer $G$, et al. European experience of 200 cases treated with botulinum-A toxin injections into the detrusor muscle for urinary incontinence due to neurogenic detrusor overactivity. Eur Urol 2004;45:510-5.

4. Sahai A, Khan MS, Dasgupta P. Efficacy of botulinum toxin-A for treating idiopathic detrusor overactivity: results from a single center, randomized, double-blind, placebo controlled trial. J Urol 2007;177:2231-6.

5. Schmid DM, Sauermann MW, Schuessler B, et al. Experience with 100 cases treated with botulinum-A toxin injections in the detrusor muscle for idiopathic overactive bladder syndrome refractory to anticholinergics. J Urol 2006; 176:177-85.

6. Herschorn S. Gajewski J, Ethans K, et al. Efficacy of botulinum toxin A injection for neurogenic detrusor overactivity and urinary incontinence: a randomized, double-blind trial. J Urol; In press.

7. Brubaker L, Richter HE, Visco A, et al. Refractory idiopathic urge urinary incontinence and botulinum $A$ injection. J Urol 2008;180:217-22.

8. Karsenty $G$, Denys $P$, Amarenco $G$, et al. Botulinum toxin $A$ (Botox) intradetrusor injections in adults with neurogenic detrusor overactivity/neurogenic overactive bladder: a systematic literature review. Eur Urol 2008;53:275-87.

9. Kuo HC, Liao CH, Chung SD. Adverse events of intravesical botulinum toxin A injections for idiopathic detrusor overactivity: risk factors and influence on treatment outcome. Eur Urol 2010;Sep 17 Epub.

10. Dmochowski R, Chapple C, Nititi VW, et al. Efficacy and safety of onabotulinumtoxin A for idiopathic overactive bladder: a double-blind, placebo controlled, randomized, dose ranging trial. J Urol 2010; 184:2416-22.

11. Dowson C, Khan MS, Dasgupta P, et al. Repeat botulinum toxin-A injections for treatment of adult detrusor overactivity. Urology 2010;7:661-7.

12. Naumann $M$, Carruthers $A$, Carruthers J, et al. Meta-analysis of neutralizing antibody conversion with onabotulinumtoxin A (Botox) across multiple indications. Movement Disorders 2010;25:2211-8.

13. Wu JM, Siddiqui NY, Amundsen CL, et al. Cost-effectiveness of botulinum toxin A versus anticholinergic medications for idiopathic urge incontinence. J Urol 2009;181:2181-6.

14. Kalsi V, Popat RB, Apostolidis A, et al. Cost-consequence analysis evaluating the use of botulinum neurotoxin-A in patients with detrusor overactivity based on clinical outcomes observed at a single UK centre. Eur Urol 2006:49:519-27.

Correspondence: Dr. Lesley Carr, Department of Surgery, University of Toronto, Sunnybrook Health Sciences Centre, Toronto ON M4N 3M5; Lesley.Car@@sunnybrook.ca 


\title{
Moving beyond ineffective medication for $\mathrm{OAB}$
}

\author{
Stephen S. Steele, MD, FRCSC
}

Assistant Professor, Departments of Urology and Pediatrics, Queen's University, Kingston, ON

I $t$ is intriguing when reading articles extolling the virtues of anticholinergic medication in the treatment of overactive bladder $(\mathrm{OAB})$ that very little time is spent exploring the issue of quality of life which, in essence, is the major complaint of this patient population. ${ }^{1}$ Unfortunately, compliance to anticholinergic medication secondary to side effects and poor patient perceived benefit in symptom reduction are extremely poor. Current data document medication adherence rates of less than $35 \% .^{2}$ This means two-thirds of our $\mathrm{OAB}$ patients are no better than when they initially saw us in consult and have had no improvement in their quality of life!

The data on Botulinum toxin A (BTX A) are much more encouraging. As I reported earlier, ${ }^{3}$ a recent study by Sahai and colleagues used the Kings Health Questionnaire (KHQ) and demonstrated that the KHQ subdomains were all significantly improved in patients who received BTX A compared with placebo. ${ }^{4}$ Dmochowski has reported similar statistically significant improvements. ${ }^{5}$

It is difficult to understand the resistance to BTX A. Opponents suggest that BTX A should be second- or thirdline therapy secondary to the possible risks of the procedure. The two biggest issues (distal muscle weakness and urinary retention) are definitely important possible complications. However, distal muscle weakness has never been reported post-BTX A injection for idiopathic OAB. ${ }^{6}$ This has only occurred in neurogenic $\mathrm{OAB}$ patients, which is an entirely different patient population. The rate of urinary retention is decreasing as we continue to modify and decrease our dose; even those unfortunate enough to go into retention still have a markedly improved quality of life. ${ }^{7}$

While many suggest that BTX A should be reserved for patients who have failed first-line therapy, I would suggest that failing anticholinergic medication is likely a foregone conclusion. Thus, it seems entirely appropriate that BTX A, with its statistically significant increased efficacy, improved patient quality of life and low side effect profile should be the first-line treatment for $\mathrm{OAB}$, rather than toiling on with ineffective treatment with intolerable side effects.

Competing interests: None declared.

\section{References}

1. Carr LK. Botulinum toxin A should not be first-line therapy for overactive bladder. Can Urol Assoc $\mathrm{J}$ 2011;5:204-5; D0I:10.5489/cuaj.11044.

2. Sears $\mathrm{CL}$, Lewis $\mathrm{C}$, Noel $\mathrm{K}$, et al. Overactive bladder medication adherence when medication is free to patients. J Urol 2010;183:1077-81.

3. Steele SS. Botulinum toxin A: First-line therapy for idiopathic detrusor overactivity. Can Urol Assoc J 2011;5:207-9; D01:10.5489/cuaj.11045.

4. Sahai A, Dowson C, Khan MS, et al. Improvement in quality of life after botulinum toxin-A injections for idiopathic detrusor overactivity: results from a randomized double blind placebo-controlled trial. BJU Int 2009; 103(3):1509-15.

5. Dmochowski R, Chapple C, Nitit VW, et al. Efficacy and safety of OnabotulinumtoxinA for idiopathic overactive bladder: A double-blind, placebo controlled, randomized, dose ranging trial. J Urol 2010;184:2416-22.

6. Sahai A, Dowson C, Khan MS, et al. Repeat botulinum toxin-A injections for treatment of adult detrusor overactivity. Urology 2010;7:661-7.

7. Kessler TM, Khan S, Panicker J, et al. Clean intermittent self-catheterization after botulinum neurotoxin A injection: Short-term effect on quality of life. Obstet Gynecol 2009;113:1046-51.

Correspondence: Dr. Stephen S. Steele, 76 Stuart St, Kingston, 0N K7L 2V7; steeles@kgh.kari.net 


\title{
POINT-COUNTERPOINT
}

\section{Botulinum toxin A: First-line therapy for idiopathic detrusor overactivity}

\author{
Stephen S. Steele, MD, FRCSC
}

Assistant Professor, Departments of Urology and Pediatrics, Queen's University, Kingston, ON

Cite as: Can Urol Assoc J 201 1;5(3):207-9; D01:10.5489/cuaj.11045

W e are well aware that overactive bladder $(\mathrm{OAB})$ is extremely common. The most recent Canadian bladder survey by Herschorn and colleagues demonstrated that $\mathrm{OAB}$ symptoms were present in $13.1 \%$ of men and $14.7 \%$ of women across Canada. ${ }^{1}$ In addition, it has been shown to have a tremendous impact on a patient's quality of life, similar to that experienced by someone with diabetes or rheumatoid arthritis. ${ }^{2,3}$

Unfortunately, the effectiveness of current $O A B$ treatment protocols in alleviating patient symptoms are not adequate, which is why investigators are looking at new agents, such as BTX A. Currently, there are over 10 different anticholinergic medications on the Canadian market for OAB. The most commonly prescribed medication for this condition, oxybutynin, is fraught with potential problems. The FDA, in 2007, released a warning; this medication is to be used with caution in the elderly and children and common side effects are hallucinations, confusion and cognitive changes. ${ }^{4}$ Others researchers, such as Kay and colleagues, have documented the potential for serious cognitive decline with this medication. ${ }^{5}$

As a result, urologists have switched to treating $O A B$ with newer medications to avoid these neurological sequelae. The drawback with these new medications, unfortunately, still revolves around side effects and, ultimately, patient compliance. The significant side effects of dry mouth and constipation, as well as poor patient perceived benefit in symptom reduction, have resulted in extremely poor medication adherence rates. Sears and colleagues examined anticholinergic medication compliance in a health care system in which medication was given free to patients; the study demonstrated an adherence rate of only $34 \%{ }^{6}{ }^{6}$ This is similar to earlier research by Shaya and colleagues, which demonstrated only a $32 \%$ adherence rate. $^{7}$

Secondary to these significant side effects and poor patient compliance, investigators have searched for a bet- ter alternative for treating $\mathrm{OAB}$. Urologists were already familiar with BTX A, having used it in a variety of other urologic disorders, such as ureteral stent colic, ${ }^{8}$ interstitial cystitis, ${ }^{9,10}$ prostatitis $^{10}$ and benign prostatic hyperplasia. ${ }^{10,11}$ As data emerged regarding its effectiveness to treat neurogenic detrusor overactivity, ${ }^{12}$ it was only natural to consider its use to treat non-neurogenic detrusor overactivity (OAB). Many urologists believe it is a matter of time before BTX A becomes an integral cog in the treatment algorithim for OAB. This change in treatment strategy is fuelled by the inability of the current treatment regimen, primarily anticholinergics, to adequately alleviate symptoms in $\mathrm{OAB}$ patients.

Current work has established the effectiveness of BTX A in $\mathrm{OAB}$. Sahia and colleagues, ${ }^{13}$ in a randomized, double-blind placebo control study, demonstrated statistically significant increases in cystometric capacity and reduced frequency, urgency urinary incontinence and urgency with the injection of 200 units of BTX A. The beneficial effects were maintained for at least 24 weeks which, incidentally, is longer than most people stay on anticholinergic medication. Others researchers have demonstrated similar statistically significant amelioration of symptoms with BTX A. ${ }^{14-16}$

However, with any "new" procedure, patient safety is always extremely important. Detractors of the use of BTX A will express concern regarding urinary retention, urinary tract infections (UTIs), hematuria, distant muscle weakness, as well as the invasiveness of the procedure. There is no doubt that these are legitimate concerns if, in fact, they are truly relevant.

It has been clearly demonstrated that BTX A can easily and comfortably be given under local anesthetic in the cystoscopy suite in a matter of minutes with minimal discomfort to the patient and with minimal side effects. ${ }^{17}$ Hematuria does occur, but to date there is not a single case report of an individual requiring a blood transfusion post-BTX A injection. Urinary tract infections do occur ${ }^{16,18}$ in the setting of BTX A injection, but there is no data demonstrating an increased risk over cystoscopy alone. 
Distant muscle weakness has definitely been described with BTX A injections. However, the frequency is extremely low (about 20 reported cases in the literature). ${ }^{18}$ None of these cases required any intervention and all reports were described in patients with neurogenic detrusor overactivity. Secondary to the small incidence (less than $1 \%$ ), the association and causation of BTX A in precipitating these events has not been well-established. ${ }^{18}$

What has been well-established is the risk of urinary retention with BTX A injections. ${ }^{13-16,18}$ The risk has been shown to be dramatically diminished with decreasing doses of BTX A. ${ }^{19}$ However, even for individuals who were unfortunate enough to go into retention, researchers have demonstrated that, in properly counselled individuals, quality of life still markedly improved versus before the procedure. ${ }^{20}$ In fact, quality of life has been extensively studied with regards to BTX A, and, not surprisingly, patients are extremely satisfied with the procedure. A recent study by Sahai and colleagues used the Kings Health Questionnaire (KHQ), which included the following subdomains to measure quality of life: "incontinence impact," "emotions," "physical limitations," "social limitations" and "severity measures." These subdomains significantly improved in patients who received BTX-A compared with placebo. ${ }^{21}$

Drug costs in the Canadian health care system will always be a major obstacle. Determining the cost-effectiveness of an intervention, therefore, is a critical undertaking and is more informative than a strict comparison of drug costs. Wu and colleagues assessed the cost-effectiveness of BTX A versus anticholinergic medications for idiopathic urge incontinence. ${ }^{22}$ The authors discovered that BTX A was much more cost-effective per year than anticholinergic medication. In fact, for anticholinergics to more cost-effective than BTX A injections, the compliance with anticholinergics would have to exceed $75 \%$. Alternatively, the injection costs would have to more than double.

The final area of concern for opponents of BTX A involves the possibility of damage to the bladder mucosa with repeated injections every 6 to 9 months. Although the data on this topic are not as robust as that on amelioration of symptoms and quality of life, they are still reassuring. Apostolidis and colleagues biopsied the injection sites and found that BTX A did not appear to be producing significant inflammatory changes, fibrosis or dysplastic changes in urothelium. ${ }^{23}$

Digesting this plethora of information obviously begs the question: should BTX A be used as a first-line treatment for patients suffering from non-neurogenic detrusor overactivity? Do we continue to use inferior agents with serious significant side effects, poor patient compliance and low success rates? I and others would suggest that the usefulness and success of BTX A make it an excellent candidate to supplant anticholinergics as first-line treatment for OAB.
In fact, a recent European Consensus panel reviewed the literature concerning BTX A in the management of neurogenic and non-neurogenic detrusor overactivity; the panel gave a Grade A recommendation to BTX A that it was a safe and effective. ${ }^{24}$ There are still a few issues that require clarification, most notably the appropriate dose that balances side effects with symptom benefits. Current research places this dose between 100 and 150 units. $^{19}$

Dose notwithstanding, the age of BTX A for OAB is upon us. Perhaps it is time to stop delaying the inevitable and take the step into the 21 st century instead of continuing with old treatment regimens fraught with poor compliance, intolerable side effects and poor cost-effectiveness.

Competing interests: None declared.

\section{References}

1. Herschorn S, Gajewski J, Shultz J, et al. A population-based study of urinary symptoms and incontinence: the Canadian Urinary Bladder Survey. BJU Int 2008;101:52-8.

2. $O^{\prime}$ Connor RM, Johannesson, $M$, Hass $S L$, et al. Urge incontinence. Quality of life and patients' valuation of symptom reduction. Pharmacoeconomics 1998; 14:531-9.

3. Komaroff AL, Fagioli LR, Doolittle TH, et al. Health status in patients with chronic fatigue syndrome an in general population and disease comparison groups. Am J Med 1996;101:281-90.

4. U.S. Food and Drug Administration Warning, U.S. Department of Health and Human Services, April, 2007.

5. Kay G, Crook T, Rekeda L, et al. Differential effects of the antimuscarinic agents darifenacin and oxybutynin ER on memory in older subjects. Eur Urol 2006:50:317-26.

6. Sears $\mathrm{CL}$, Lewis $\mathrm{C}$, Noel $\mathrm{K}$, et al. Overactive bladder medication adherence when medication is free to patients. J Urol 2010;183:1077-81.

7. Shaya FT, Blume S, Gu A, et al. Persistence with overactive bladder pharmacotherapy in a Medicaid population. Am J Manag Care 2005;11:S121-9.

8. Gupta $M$, Patel $\mathrm{T}$, Xavier $\mathrm{K}$, et al. Prospective randomized evaluation of periureteral botulinum toxin type A injection for ureteral stent pain reduction. J Urol 2010;183:598-602.

9. Pinto R, Lopes $T$, Frias $B$, et al. Trigonal injection of botulinum toxin $A$ in patients with refractory bladder pain syndrome/intersititial cystitis. Eur Urol 2010;58:360-5.

10. Smith CP, Radziszewski P, Borkowski A, et al. Botulinum toxin A has antinociceptive effects in treating intersititial cystitis. Urology 2004;64:871-5.

11. Brisinda $G$, Cadeddu $F$, Vanella $S$, et al. Relief by botulinum toxin of lower urinary tract symptoms owing to benign prostatic hyperplasia: early and long-term results. Urology 2009;73:90-4.

12. Schurch $B$, Seze $M$, Denys $P$, et al. Botulinum toxin type $A$ is a safe and effective treatment for neurogenic urinary incontinence: Results of a single treatment, randomized placebo controlled 6-month study. J Urol 2005:174:196-200

13. Sahai A, Khan MS, Dasgupta P. Efficacy of botulinum toxin-A for treating idiopathic detrusor overactivity: Results from a single center, randomized, double-blind, placebo controlled trial. J Urol 2007;177:2231-6.

14. Schmid DM, Savermann P, Werneer $M$, et al. Experience with 100 cases treated with botulinum-A toxin injections in the detrusor muscle for idiopathic overactive bladder syndrome refractory to anticholinergics. J Urol 2006;176:177-85.

15. White WM, Pickens RB, Doggweiler $R$, et al. Short-term efficacy of botulinum toxin A for refractory overactive bladder in the elderly population. J Urol 2008;180:2522-6.

16. Anger JT, Weinberg A, Suttorp MJ, et al. Outcomes of intravesical botulinum toxin for idiopathic overactive bladder symptoms: A systematic review of the literature. J Urol 2010;183:2258-64.

17. Cohen BL, Rivera R, Barboglio P, et al. Safety and tolerability of sedation-free flexible cystoscopy for intradetrusor botulinum toxin-A injection. J Urol 2007;177:1006-10.

18. Sahai A, Dowson C, Khan MS, et al. Repeat botulinum toxin-A injections for treatment of adult detrusor overactivity. Urology 2010;7:661-7.

19. Dmochowski R, Chapple C, Nitti VW, et al. Efficacy and safety of OnabotulinumtoxinA for idiopathic overactive bladder: A double-blind, placebo controlled, randomized, dose ranging trial. J Urol 2010;184:241622. 
20. Kessler $T M$, Khan S, Panicker J, et al. Clean intermittent self-catheterization after botulinum neurotoxin $A$ injection: Short-term effect on quality of life. Obstet Gynecol 2009;113:1046-51.

21. Sahai A, Dowson C, Khan MS, et al. Improvement in quality of life after botulinum toxin-A injections for idiopathic detrusor overactivity: results from a randomized double blind placebo-controlled trial. BJU Int 2009;103:1509-15.

22. Wu JM, Siddiqui NY, Amundsen CL, et al. Cost effectiveness of botulinum toxin A versus anticholinergic medications for idiopathic urge incontinence. J Urol 2009;181:2181-6.

23. Apostolidis A, Jacques TS, Freeman A, et al. Histological changes in the urothelium and suburolthelium of human overactive bladder following intradetrusor iniections of botulinum neurotoxin A for the treatment of neurogenic or idiopathic detrusor overactivity. Eur Urol 2008;53:1245-53.
24. Apostolidis A, Dasgupta $P$, Denys $P$, et al. Recommendations on the use of botulinum toxin in the treatment of lower urinary tract disorders and pelvic floor dysfunctions: A European consensus report. Eur Urol 2009:55:100-19.

Correspondence: Dr. Stephen S. Steele, 76 Stuart St, Kingston, 0N K7L 2V7; steeles@kgh.kari.net

\section{More data are needed to use BTX A as first-line treatment}

\section{Lesley K. Carr, MD, FRCSC}

Assistant Professor, Department of Surgery, University of Toronto, Sunnybrook Health Sciences Centre, Toronto, ON

Cite as: Can Urol Assoc J 201 1;5 (3):209; D01:10.5489/cuaj.11082

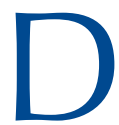

r. Steele succinctly summarizes the efficacy and safety of Botulinum toxin A (BTX A) - a point which is not in debate. ${ }^{1}$ However, the use of BTX $A$ as a primary modality of treatment for $O A B$ has never been reported. While it is likely that BTX A will be able to control overactivity in anticholinergic naïve patients, it is unclear what side effects, such as urinary retention, will be seen in such a group. Research into patient preferences and decision-making as it relates to the weighted value of the invasiveness of intervention and side effects balanced with overall levels of improvement in symptoms would yield insight. A randomized trial between BTX A and an anticholinergic medication in patients with moderate to severe $\mathrm{OAB}$ with detrusor overactivity who are naïve to treatment may be reasonable to address this issue, especially in the subset of neurogenic $\mathrm{OAB}$ who are already intermittently catheterizing.
For the time being, there is no data to support the use of BTX $A$ as a primary treatment for OAB. The long-term success beyond 10 years of BTX A for OAB is unknown. Thus, patients should continue to be treated in a stepwise fashion beginning with lifestyle and behavioural changes, and oral pharmacotherapy.

Competing interests: None declared.

\section{Reference}

1. Steele SS. Botulinum toxin A: First-line therapy for idiopathic detrusor overactivity. Can Urol Assoc J 2011;5:207-9; D01:10.5489/cuaj.11045

Correspondence: Dr. Lesley Carr, Department of Surgery, University of Toronto, Sunnybrook Health Sciences Centre, Toronto ON M4N 3M5; Lesley.Car@@sunnybrook.ca 